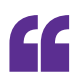

tumour cell entry into the lymph node requires active cell migration
METASTASIS

\section{Active lymph nodes}

Lymph node metastases are indicative of poor prognosis but the mechanisms of tumour cell dissemination via the lymphatics are poorly understood. Although it is widely believed that tumour cells enter the lymph nodes passively with the flow of lymph, previous data have indicated that some chemokines may promote lymphatic extravasation and metastasis. Das et al. now demonstrate that tumour cell entry into the lymph node requires active cell migration and they also identify the lymphatic endothelium of the lymph node subcapsular sinus as a critical gatekeeper.

The authors investigated whether conditioned media from cultured lymphatic endothelial cells (LECs) was chemotactic for several metastatic cancer cell lines. The conditioned media induced the migration of metastatic melanoma cells (and not poorly metastatic melanoma cells) and this could be attributed to the chemokine CCL1. CCR8, a G protein-coupled receptor (GPCR), is activated by CCL1, and the authors found that the cell lines that responded to LEC-conditioned media expressed CCR8, and that chemotaxis was inhibited when the cells were treated with MC148 (a CCR8 antagonist) or with blocking antibodies to CCL1. Further experiments confirmed that LEC-derived
CCL1 activates CCR8-mediated intracellular signalling in tumour cells, which resulted in cellular changes that are consistent with cell migration.

Is the CCR8-CCL1 paracrine pathway important in lymphatic metastasis? The suppression of CCR8 expression or activity in human melanoma cells did not affect tumour growth or vascularization on implantation into immunodeficient mice, but significantly reduced the incidence of lymph node metastasis. Furthermore, CCL1 was not detected in the tumour microenvironment but was detected in the lymphatic endothelium of the lymph node subcapsular sinus. Further investigations in vivo revealed that CCR8 activation in tumour cells was required for tumour cell extravasation from lymphatic vessels, specifically for the transmigration of tumour cells from the subcapsular sinus into the lymph node cortex.

These data unpick the process of lymphatic dissemination and identify the sequence of steps leading to lymph node metastasis. The authors showed that CCR8 is expressed by a large subset of human melanoma samples, and it will be interesting to determine whether this pathway can be targeted to prevent lymph node metastasis.

Gemma K. Alderton

ORIGINAL RESEARCH PAPER Das, S. et al.

Tumor cell entry into the lymph node is controlled by CCL1 chemokine expressed by lymph node lymphatic sinuses. J. Exp. Med. 210, 1509-1528 (2013)

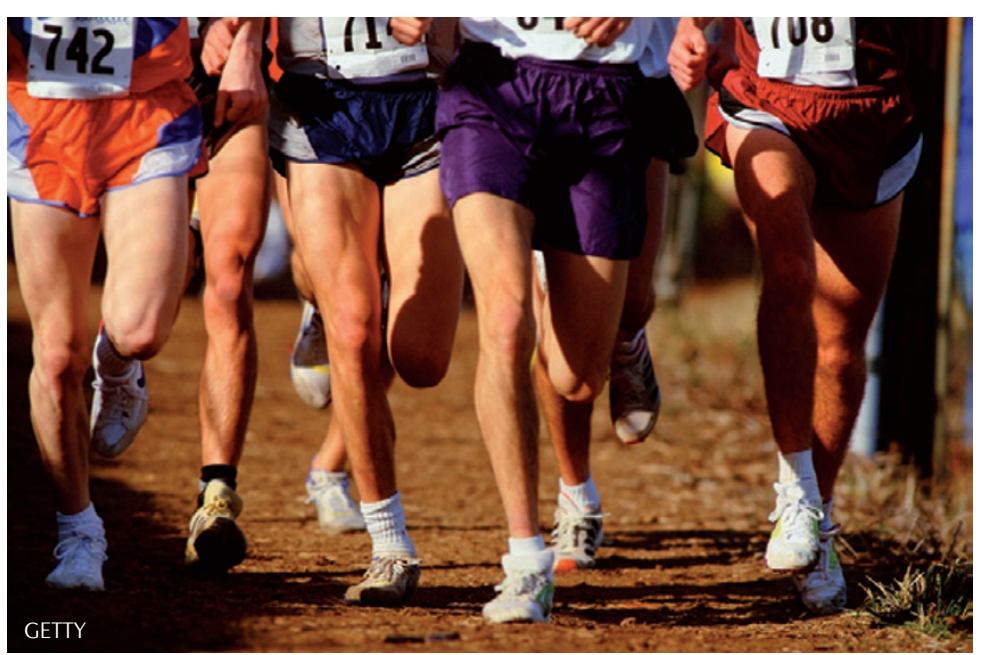

